\title{
Usefulness of Pseudocontinuous Arterial Spin-Labeling for the Assessment of Patients with Head and Neck Squamous Cell Carcinoma by Measuring Tumor Blood Flow in the Pretreatment and Early Treatment Period
}

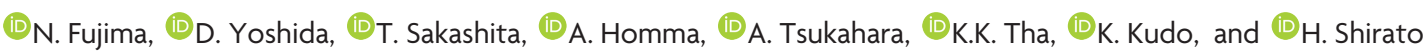

\begin{abstract}
BACKGROUND AND PURPOSE: For the assessment of the treatment response in non-surgical treatment, tumor blood flow provides the functional information of the tumor which is different from the morphological information such as tumor volume. The purpose of this study was to evaluate the diagnostic value of tumor blood flow values obtained by pseudocontinuous arterial spin-labeling in patients with head and neck squamous cell carcinoma.
\end{abstract}

MATERIALS AND METHODS: Forty-one patients with head and neck squamous cell carcinoma were evaluated by using pseudocontinuous arterial spin-labeling. Quantitative tumor blood flow was calculated at the pretreatment and the early treatment periods in all the patients, and the percentage change of tumor blood flow between the two was calculated. At the early treatment period, based on their tumor volume reduction rate, we divided the patients into stable disease and partial response groups for a subgroup analysis. The local control or failure was confirmed either by histopathology or by radiologic evaluation within the follow-up.

RESULTS: Pretreatment tumor blood flow in patients in the failure group was significantly lower than that in patients in the local control group. In the subgroup analysis of patients with stable disease, the percentage change of tumor blood flow was significantly larger (due to the tumor blood flow increase from pretreatment value) in the local control group than in the failure group. In addition, in patients with a partial response, the percentage change of tumor blood flow was significantly smaller (due to the tumor blood flow decrease from the pretreatment value) in the local control group than in the failure group. The accuracy for determination of the local control group or the failure group in pretreatment tumor blood flow was 0.83 and that in the combination use of the percentage change of tumor blood flow and tumor volume in the early treatment period was 0.93 .

CONCLUSIONS: Tumor blood flow obtained by pseudocontinuous arterial spin-labeling can be useful for the determination of local control. The combined use of the percentage change of tumor blood flow and tumor volume had particularly high diagnostic accuracy.

ABBREVIATIONS: $\mathrm{HNSCC}=$ head and neck squamous cell carcinoma; $\mathrm{PCASL}=$ pseudocontinuous arterial spin-labeling; $\mathrm{PR}=$ partial response; $\mathrm{SCC}=$ squamous cell carcinoma; $\mathrm{SD}=$ stable disease; $\mathrm{TBF}=$ tumor blood flow; $\mathrm{TV}=$ tumor volume

N onsurgical, organ-preserving treatments, including chemotherapy, radiation therapy, and combinations of these approaches, are important therapeutic modalities for patients with

Received May 6, 2015; accepted after revision July 6

From the Department of Diagnostic and Interventional Radiology (N.F., D.Y., A.T., K.K.), Hokkaido University Hospital, Sapporo, Japan; Departments of OtolaryngologyHead and Neck Surgery (T.S., A.H.) and Radiation Medicine (K.K.T., H.S.), Hokkaido University Graduate School of Medicine, Sapporo, Japan; and Global Station for Quantum Medical Science and Engineering (K.K.T., H.S.), Global Institution for Collaborative Research and Education, Sapporo, Japan.

This study received grant support from the Ministry of Education, Culture, Sports, Science, and Technology, Japan (ID 25861047).

Paper previously presented in part at: Joint Annual Meeting of the International Society for Magnetic Resonance in Medicine-European Society for Magnetic Resonance in Medicine and Biology, May 10-16, 2014; Milan, Italy.

Please address correspondence to Noriyuki Fujima, MD, PhD, Department of Diag nostic and Interventional Radiology, Hokkaido University Hospital, N15, W7, KitaKu, Sapporo 060-8638, Japan; e-mail: Noriyuki.Fujima@mb9.seikyou.ne.jp head and neck squamous cell carcinoma (HNSCC). ${ }^{1-3}$ Detecting early treatment response can impact and improve patient care by allowing tailoring of treatment and optimization of follow-up strategy. Early treatment responses are usually assessed by anatomic imaging with CT or MR imaging to detect morphologic changes of the tumor, as noted in the Response Evaluation Criteria in Solid Tumors rules. ${ }^{4}$ However, studies reported that morphologic changes are limited in their ability to predict treatment response, ${ }^{5,6}$ and, thus, such information alone is not always sufficient for assessing the local control or failure at the primary site. In contrast, the measurement of tumor blood flow (TBF) provides functional information because tumor perfusion reflects neovascularity or angiogenic activity, which is closely related to

\footnotetext{
- Indicates open access to non-subscribers at www.ajnr.org

http://dx.doi.org/10.3174/ajnr.A4513
} 
tumor growth. ${ }^{7}$ In patients with HNSCC, the TBF is usually assessed by a dynamic contrast-enhanced method. ${ }^{8-11}$ However, this method is somewhat invasive and requires IV placement and contrast injection, and repetitive scanning is sometimes difficult, especially during chemotherapy when renal dysfunction frequently occurs (approximately $25 \%-42 \%$ ) in patients given cisplatin. ${ }^{12,13}$ Because of the difficulties of repetitive scanning, there have been few studies of the changes in TBF with repetitive scanning over the treatment period, and the number of patients in these reports is quite small, at 10 to $<20$ patients. ${ }^{14-16}$ Thus, the role and potential of TBF measurement during treatment remain unclear. TBF measurement by pseudocontinuous arterial spinlabeling (pCASL), which was introduced for the noninvasive measurement of tissue blood flow without the need for a contrast agent and with no radiation exposure, ${ }^{17,18}$ was recently demonstrated to be feasible and applicable for HNSCCs ${ }^{19}$; pCASL thus has potential for the evaluation of treatment effects in patients with HNSCC without the risks with repetitive scanning of contrast-induced nephropathy during chemotherapy and increased radiation exposure. The purpose of the present study was to evaluate the diagnostic value of TBF assessed by pCASL for the determination of local control or failure in patients with HNSCC.

\section{MATERIALS AND METHODS \\ Patients}

The study protocol was approved by our institutional review board, and written informed consent was obtained from all the patients. From September 2010 to August 2013, 45 consecutive patients who were referred to our hospital to receive superselective arterial infusions of cisplatin with concomitant radiation therapy for a diagnosis of nasal, sinonasal, or oropharynx cancer were enrolled in this prospective study with the following inclusion criteria: 1) histopathologic diagnosis of HNSCC, and 2) plan for a full course of curative treatment with a radiation dose of 70 Gy. In all the patients, MR scanning, including pCASL, was performed in both the pretreatment and early treatment period, as described below. Among these 45 patients, 4 discontinued treatment due to severe complications and were excluded. A total of 41 patients, therefore, were considered eligible for this study: 36 men (mean age, 61.2 years; range, $46-77$ years) and 5 women (mean age, 57.4 years; range $43-69$ years). The histopathologic diagnoses were squamous cell carcinoma (SCC) in all the patients. The primary lesions of the 41 patients, detail of histopathologic information, and T stage were as follows: nasal or sinonasal cavity in 24 patients (well differentiated in 3 , moderate in 10 , poor in 11 ; keratinizing type in 9 , nonkeratinizing type in 15 ; T1/2 in 1 , T3 in 8 , T4a in 11, and T4b in 4) and oropharynx in 17 patients (well differentiated in 3, moderate in 11 , poor in 3; keratinizing type in 12 , nonkeratinizing type in 5 ; T1/2 in 4 , T3 in 1 , T4a in 9 , and T4b in 3). The treatment regimen was superselective arterial infusions of cisplatin with concomitant radiation therapy for all the patients. Treatment details were as follows: arterial infusion of cisplatin $\left(100-120 \mathrm{mg} / \mathrm{m}^{2}\right.$ per wk for 4 weeks) to the dominant blood supply of the primary tumor by using a microcatheter, with concurrent radiation therapy of a total of 70 Gy in 35 fractions. MR imaging scans, including pCASL, were performed in all the patients before treatment and again in the early treatment period.
The time intervals between the pretreatment pCASL and the beginning of the treatment ranged from 1 to 25 days (mean, 9.2 days). MR scanning in the early treatment period was performed on delivery of 16-20 Gy (mean, $19.1 \mathrm{~Gy}$ ) of the total $70 \mathrm{~Gy}$, which occurred just after the second arterial infusion of cisplatin.

\section{Clinical End Point}

For all the patients, clinical and radiologic follow-ups were performed after the treatment to determine the final diagnosis of failure or local control at the primary site. After treatment or during follow-up, based on the patients' radiologic findings: 1) surgical resection was performed in the patients in whom a residual mass lesion was suspected, and 2) a biopsy was performed in the patients with thickened posttreatment granulation tissue. In addition, careful follow-up was performed in the patients with no mass lesion and/or granulation tissue. Local failure at the primary site was determined by histopathologic confirmation of HNSCC by biopsy specimen or surgical resection, or by enlargement of residual tumor during follow-up. Local control at the primary site was determined by histopathologic confirmation of the absence of HNSCC by surgical resection, the absence of enlargement of the possible residual tumor, or the absence of a new lesion in the posttreatment granulation tissue within the follow-up period, which was at least 1 year.

\section{MR Imaging Protocol}

All MR imaging was performed by using a 3T unit (Achieva TX; Philips Healthcare, Best, the Netherlands) with a 16-channel neurovascular coil. First, conventional MR images were obtained to evaluate the primary tumor. These images included 1) axial T1WI with a spin-echo sequence (TR, 450 milliseconds; TE, 10 milliseconds; FOV, $240 \times 240 \mathrm{~mm} ; 512 \times 512$ matrix; section thickness, $5 \mathrm{~mm}$; intersection gap, 30\%; scanning time, 2 minutes and 12 seconds), and 2) axial T2WI with a TSE sequence with fat suppression (TR, 4500 milliseconds; TE, 70 milliseconds; TSE factor, 9; FOV, $240 \times 240 \mathrm{~mm}$; $512 \times 512$ matrix; section thickness, 5 $\mathrm{mm}$; intersection gap, 30\%; scanning time, 2 minutes and 6 seconds).

In the pCASL scanning, coronal T2WI was performed to obtain anatomic localization of the carotid artery for positioning of the labeling slab. A T1 map was also obtained to measure the longitudinal relaxation in the tumor tissue, and the map was used for the TBF quantification. The acquisition of pCASL was performed by using multishot spin-echo echo-planar imaging to obtain control and labeled images. The labeling slab was placed just under the bifurcation of the internal and external carotid arteries by using coronal T2WI as a reference for the labeling of arterial water. The control images were obtained without the labeling of arterial water by using the same imaging scheme of the labeled images. The MR parameters of the pCASL were as follows: labeling duration, 1650 milliseconds; postlabel delay, 1280 milliseconds; TR, 3619 milliseconds; TE, 18 milliseconds; flip angle, 90; number of shots, 2 ; FOV , $230 \times 230 \mathrm{~mm}$; matrix, $80 \times 80$; section thickness, $5 \mathrm{~mm}$; number of sections, 15 ; acceleration factor for parallel imaging, 2; scanning time, 5 minutes and 11 seconds. All the sections were placed in parallel with the anterior/posterior commissure line. Each section was carefully placed at the same 
level in the scans taken at the pretreatment and early treatment period by using the sagittal scout image. The patients were instructed not to swallow, move their tongue, open their mouth, or make any other voluntary motion during the pCASL scan.

The coronal T2WI was obtained by TSE sequence with the following parameters: TR, 4500 milliseconds; TE, 70 milliseconds; TSE factor, 9; FOV, $240 \times 240 \mathrm{~mm}$; matrix, $512 \times 512$; section thickness, $4 \mathrm{~mm}$; intersection gap, 30\%; scanning time, 2 minutes and 6 seconds. For the T1 map, we used a gradient-echo sequence with Look-Locker readout by constant flip angle with the following parameters: TR, 7 milliseconds; TE, 1.7 milliseconds; flip angle, $7^{\circ}$; FOV , $230 \times 230 \mathrm{~mm}$; matrix, $256 \times 256$; section thickness, $10 \mathrm{~mm}$ (single section acquisition); scanning time, 6 seconds.

\section{Data Analysis}

TBF Calculation by PCASL. We calculated the TBF of the pCASL ( $f$ ) from the signal difference $(\Delta \mathrm{M})$, which was calculated by subtracting the labeled image from the control image, by using the previously described equation ${ }^{20}$ :

$$
f=\frac{\Delta M \lambda R_{1 a} \exp \left(\omega R_{1 a}\right)}{2 M_{0} \alpha}\left[1-\exp \left(-\gamma R_{1 a}\right)\right]^{-1}
$$

in which $R_{1 a}$ is the longitudinal relaxation rate of blood $(0.67$ seconds $\left.{ }^{-1}\right), \gamma$ is the labeling time ( 1.65 seconds), $\omega$ is the postlabeling delay time (1.28 seconds), $\alpha$ is the labeling efficiency (0.85), and $\lambda$ is the blood/tumor-tissue water partition coefficient (1.0 $\mathrm{g} / \mathrm{mL}){ }^{21,22} M_{0}$ is the equilibrium magnetization of the tumor tissue, which was estimated from the signal intensity of the control image and the tumor longitudinal relaxation rate obtained with the T1 map. By using Equation 1, we created the TBF maps on a pixel-by-pixel basis. We used mathematical software (Matlab version 2012a; MathWorks, Natick, Massachusetts) to calculate the TBF values for both pCASLs.

Tumor ROI Delineation. Each patient's primary tumor was outlined by a board-certified neuroradiologist (A.T.) with 18 years of experience. The delineation was performed on the axial T2WI with a polygonal ROI, and the ROI was then copied onto a TBF map (Fig 1). ROI delineation was performed by using ImageJ software (National Institutes of Health, Bethesda, Maryland) to include the soft-tissue mass (and to exclude the normal or inflammatory tissue) in the ROI obtained from the T2WI findings. T1WI was also used as a guide to determine the ROI. To avoid vascular artifacts in the ROI, the area of the vessel signal void was also delineated on the T2WI, and this area was excluded from TBF measurement. Any strong high signal area with T2WI that indicated necrosis was also excluded. The TBF value of pCASL in each patient was determined as the mean of the TBF values in the delineated ROI. If the tumor extended into 2 or more sections on the TBF map, then the mean TBF of all pixels in all ROIs of the tumor was calculated as the TBF value. The percentage change of TBF between the pretreatment and early treatment period was calculated as follows: percentage change of TBF $=100 \times(\mathrm{TBF}$ at the early treatment period)/(pretreatment TBF) -100 .

Tumor Volume Measurement. Each tumor ROI used in the TBF analysis was also used for measurement of the tumor volume (TV). The TV was calculated as follows: $[\mathrm{TV}]=($ size of ROI $) \times$
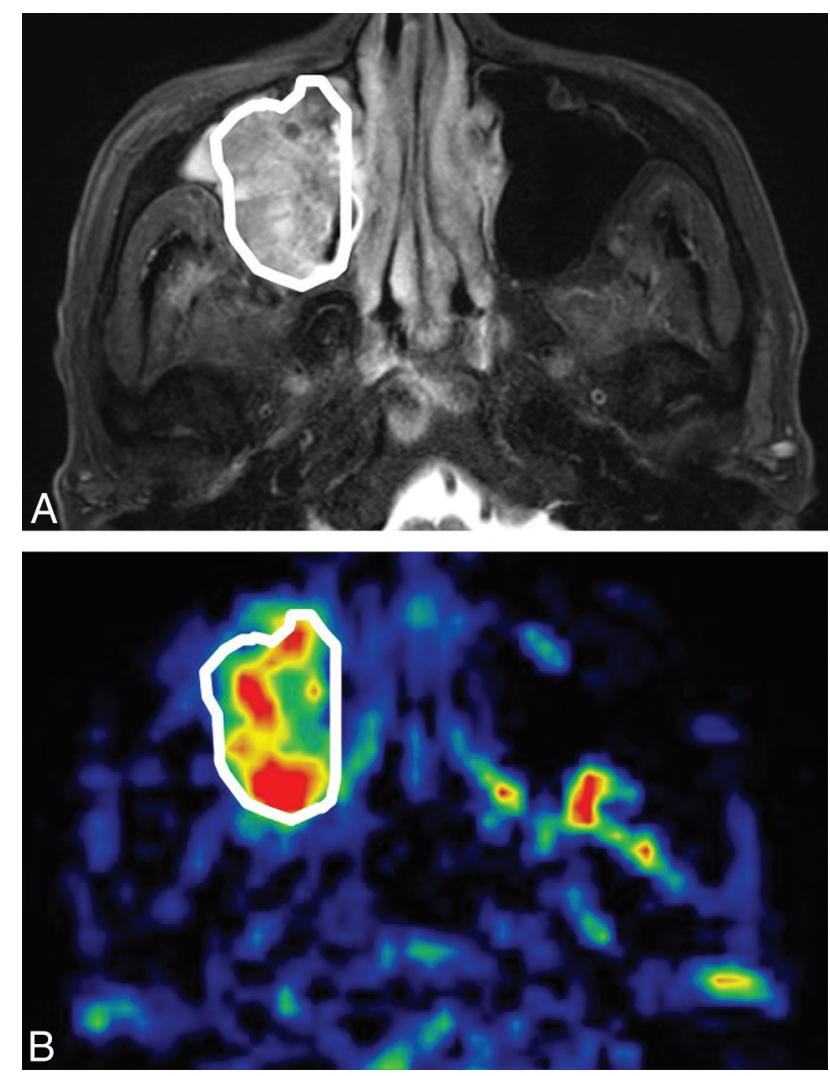

FIG 1. Tumor ROI delineation. An example of the tumor ROI delineation (a 68-year-old man with right maxillary cancer). $A$, The tumor was delineated on the axial T2WI with a polygonal ROI. B, After the delineation, the ROI was copied to the corresponding TBF map.

(section thickness). If the tumor extended beyond 2 sections, then the sum of the TVs in all the sections was calculated. The percentage change of TV between the pretreatment and early treatment periods was also calculated as follows: percentage change of TV = $100 \times(\mathrm{TV}$ at the early treatment period $) /($ pretreatment TV) 100. For the subgroup analysis, we divided the 41 patients into 2 groups based on the Response Evaluation Criteria in Solid Tumors criteria by using their TV values in the pretreatment and early treatment periods as follows: patients whose percentage change of TV reduction between the pretreatment and early treatment period was $\geq 30 \%$ (ie, percentage change of a TV of $-30 \%$ or less) made up the partial response (PR) group, and those whose percentage change of TV reduction was $<30 \%$ (percentage change of TV of more than $-30 \%$ ) formed the stable disease (SD) group. Although the diameter of the tumor's long axis at the primary site was used for the size evaluation along with Response Evaluation Criteria in Solid Tumors criteria, this value is sometimes unreliable in head and neck tumors because of their complicated shapes, and, therefore, we used the TV for the size evaluation in the present study.

\section{Statistical Analysis}

A Mann-Whitney $U$ test was used to compare pretreatment TBF and TV values between the local control and the failure groups. The same test was used to compare the percentage changes of TBF and TV in the early treatment period between the local control and the failure groups. The subgroup analysis of the percentage 
change of TBF was performed by dividing the patients according to TV change in the early treatment period into PR and SD groups. The percentage change of TBF was compared between the patients in the local control group and in the failure group by using a Mann-Whitney $U$ test in the PR and SD groups, respectively. If a significant difference was observed, then receiver operating characteristic curves were constructed for the calculation of the area under the curve and for the determination of the best diagnostic accuracy by using the closest point to the upper left corner of the receiver operating characteristic curve in the division of the local control and failure groups.

In addition, to investigate the relationship between TBF based on the location of each primary lesion and the result of the local control group or the failure group, the patients were divided into the group of patients with a lesion in the nasal or sinonasal cavity $(n=24)$ and those with a lesion in the oropharynx $(n=17)$. For each primary lesion, Mann-Whitney $U$ tests were then used to compare pretreatment TBF values, the percentage change of TBF at the early treatment period, and for subgroup analysis of the patients with SD and those with PR between the local control and failure groups. $P$ values $<.05$ were accepted as significant. Statistical software (SPSS; IBM, Armonk, New York) was used for the analysis.

\section{RESULTS}

We successfully obtained pCASL images of the 41 primary tumors in both the pretreatment and early treatment periods. Among the 41 patients, 11 were found to be in the failure group ( 8 patients with nasal or sinonasal cavity SCC, T stage of T3 in 2, T4a in 4, and T4b in 2; 3 patients with oropharynx SCC, T stage of T4a in 2, and T4b in 1). Nine patients with local failure were confirmed by histopathologic findings. Two patients with local failure and 30 with local control were determined by clinical diagnosis at follow-up (mean, 26 months; range, 12-43 months).

In the analysis of all the patients, the pretreatment TBF in the 11 patients in the failure group $(109.6 \pm 28.5 \mathrm{~mL} / 100 \mathrm{~g} / \mathrm{min})$ was significantly lower than that in the 30 patients in the local control group $(142.3 \pm 34.5 \mathrm{~mL} / 100 \mathrm{~g} / \mathrm{min})(P<.01)$ (Fig 2). Although there was a tendency for larger pretreatment TV values in the failure group patients $(38.4 \pm 27.1 \mathrm{~mL})$ compared with the patients in the local control group $(24.8 \pm 19.1 \mathrm{~mL})$, the difference was not significant $(P=.15)$. In the early treatment period, there was no significant difference in the percentage change of TBF between the failure group $(-3.9 \% \pm 11.4 \%)$ and the local control group $(-15.8 \% \pm 34.1 \%)(P=.19)$ or in the TV values between the failure group $(-32.3 \% \pm 20.1 \%)$ and the local control group $(-41.0 \% \pm 21.0 \%)(P=.24)$. In the subgroup analysis, 17 patients achieved SD and 24 patients achieved only a PR, based on the percentage change in the TV values. In the subgroup analysis of patients with SD, the percentage change of TBF among the patients in the failure group $(-0.9 \% \pm 11.9 \%)$ was significantly smaller than that in the patients in the local control group $(23.0 \% \pm 17.2 \%)(P<.01)$ (Fig 3).

In addition, the subgroup analysis of patients with $P R$ revealed that the percentage change of TBF in the patients in the failure group $(-9.2 \% \pm 9.7 \%)$ was significantly larger than that in the patients in the local control group $(-35.2 \% \pm 21.0 \%)(P<.01)$,
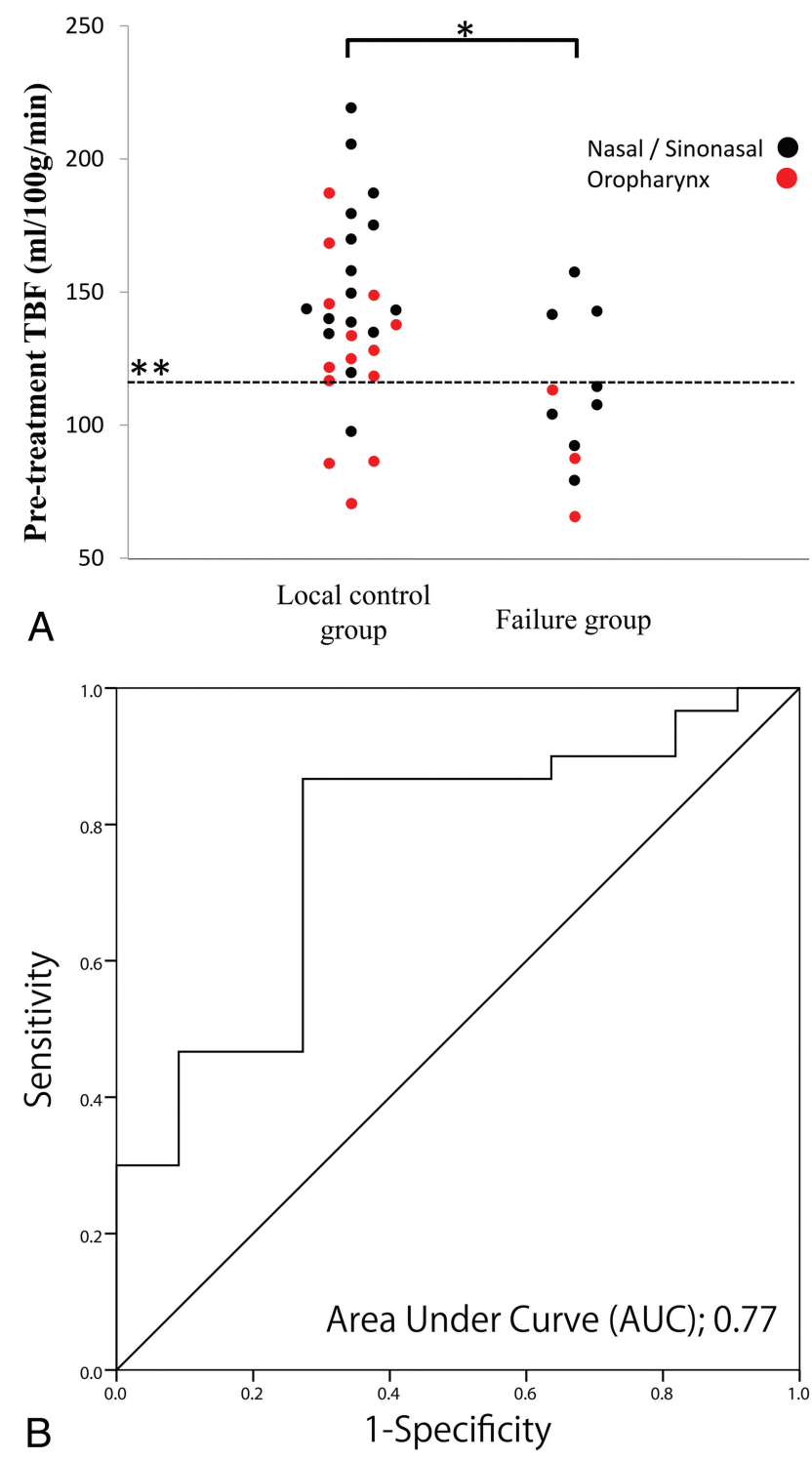

FIG 2. Pretreatment TBF in all the patients. $A$, The pretreatment TBF in patients in the failure group $(109.6 \pm 28.5 \mathrm{~mL} / 100 \mathrm{~g} / \mathrm{min})$ was significantly lower than that in patients in the local control group $(142.3 \pm 34.5 \mathrm{~mL} / 100 \mathrm{~g} / \mathrm{min})(P<.01)\left(A,{ }^{*}\right)$. $B$, The receiver operating characteristic curve analysis revealed the area under the curve of 0.77 $(P<.01)$, with the best diagnostic accuracy of 0.83 (34/41), sensitivity of $0.87(26 / 30)$, and specificity of $0.73(8 / 11)$, with a threshold value of $115 \mathrm{~mL} / 100 \mathrm{~g} / \mathrm{min}\left(A,{ }^{\star *}\right)$

which was the inverse of the trend in the SD group (Fig 3). In the receiver operating characteristic curve analysis, the best accuracy for the determination of failure or local control group in pretreatment TBF was $0.83(34 / 41)$, with a sensitivity of $0.87(26 / 30)$ and specificity of $0.73(8 / 11)$ when the threshold was set at $115 \mathrm{~mL} / 100$ $\mathrm{g} / \mathrm{min}$ (area under the curve, $0.77 ; P<.01$ ) (Fig 2). In the subgroup analysis of patients with SD, the best accuracy for the percentage change of TBF was $0.94(16 / 17)$, with a sensitivity of 1.0 $(10 / 10)$ and specificity of $0.86(6 / 7)$ when the threshold was set at $7.0 \%$ (area under the curve, $0.94 ; P<.01$ ) (Fig 3 ). In the subgroup analysis of patients with a PR, the best accuracy for the percentage change of TBF was $0.92(22 / 24)$, with a sensitivity of $0.95(19 / 20)$ and specificity of $0.75(3 / 4)$ when the threshold was set at the range of $-8.0 \%$ to $-14.0 \%$ (area under the curve, $0.89 ; P<.01$ ) 

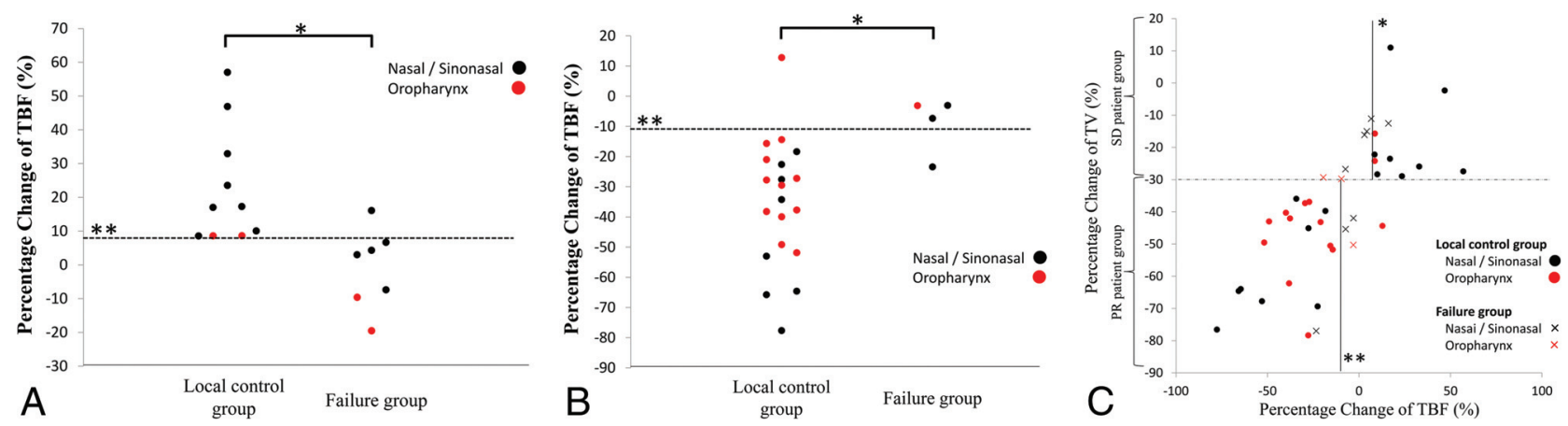

FIG 3. Percentage change of TBF in the early treatment period in the subgroup analyses of patients with SD and patients with PR. A, Subgroup analysis of patients in the SD group (the patients with $\leq 30 \%$ TV reduction in the early treatment period); the percentage change of TBF in the failure group $(-0.9 \% \pm 11.9 \%)$ was significantly lower than that in the local control group $(23.0 \% \pm 17.2 \%)(P<.01)\left({ }^{*}\right)$; the best diagnostic accuracy of $0.94(16 / 17)$ with sensitivity of $1.0(10 / 10)$ and specificity of $0.86(6 / 7)$, with the threshold value $7 \%(* \star)$ was revealed by receiver operating characteristic analysis. B, Subgroup analysis of the PR patient group (the patients with $>30 \%$ TV reduction in the early treatment period); the percentage change of TBF in the failure group $(-9.2 \% \pm 9.7 \%)$ was significantly higher than that in the local control group $(-35.1 \% \pm 21.0 \%)(P<$ $.01)(*)$. The best diagnostic accuracy of $0.92(22 / 24)$ with a sensitivity of $0.95(19 / 20)$ and specificity of $0.75(3 / 4)$, with a threshold value in the range $-8.0 \%$ to $-14.0 \%\left(^{* *}\right)$ was revealed by receiver operating characteristic analysis. $C$, Two-dimensional plot graph with the percentage change of TV on the vertical axis and the percentage change of TBF on the horizontal axis; when the threshold line was set to $7 \%(*)$ in the patients in the SD group, and the threshold was in the range $-8.0 \%$ to $-14.0 \%\left({ }^{* *}\right)$ in the PR patient group, the sensitivity, specificity, and accuracy for the division of failure and local control group were 0.97 (29/30), 0.82 (9/11), and 0.93 (38/41), respectively.

(Fig 3). When the SD and PR groups were combined in the subgroup analysis, the accuracy of the percentage change of TBF was 0.93 (38/41), with 0.97 sensitivity (29/30) and 0.82 specificity (9/ 11 ) with the setting of threshold in both $\mathrm{SD}$ and PR groups $(7.0 \%$; patients of the SD group; -8.0 to $-14.0 \%$, patients of the PR group) (Fig 3).

In the analysis based on tumor location, the pretreatment TBF values in the failure group with a nasal or sinonasal cavity lesion $(117.5 \pm 27.3 \mathrm{~mL} / 100 \mathrm{~g} / \mathrm{min})$ were significantly lower than those of the local control group $(154.3 \pm 33.4 \mathrm{~mL} / 100 \mathrm{~g} / \mathrm{min})(P<.05)$. In addition, among the patients with an oropharynx SCC, the pretreatment TBF values of the failure group $(88.8 \pm 23.8 \mathrm{~mL} / 100$ $\mathrm{g} / \mathrm{min}$ ) were significantly lower than those of the local control group $(126.7 .3 \pm 31.7 \mathrm{~mL} / 100 \mathrm{~g} / \mathrm{min})(P<.05)$. At the early treatment period, there was no significant difference in the percentage change of TBF between the failure and local control groups in both the nasal and/or sinonasal cavity SCC $(-1.4 \% \pm$ $11.8 \%$ and $-6.4 \% \pm 42.4 \% ; P=.59)$ and patients with oropharynx SCC $(-10.7 \% \pm 8.3 \%$ and $-23.0 \% \pm 21.0 \% ; P=.42)$. Among the patients with nasal and/or sinonasal cavity SCC, in the SD group ( $n=13$ : failure in 5 , local control in 8$)$, the percentage change of TBF in the failure group $(-4.5 \% \pm 8.4 \%)$ was significantly smaller than that in the local control group $(26.6 \% \pm$ $17.6 \%)(P<.05)$, and in the PR group $(n=11$ : failure in 3 , local control in 8$)$, the percentage change of TBF in the failure group $(-11.2 \% \pm 10.7 \%)$ was significantly larger than that in the local control group $(-39.4 \% \pm 32.5 \%)(P<.05)$. Among the patients with an oropharynx SCC, in the SD group $(n=4$ : failure in 2, local control in 2), the percentage change of TBF in the failure group $(-14.6 \% \pm 7.0 \%)$ tended to be smaller than that in the local control group $(8.6 \% \pm 0.03 \%)$. In addition, in the PR group $(n=$ 11: failure in 1 , local control in 10), the percentage change of TBF in the patients in the failure group $(-3.1 \%)$ tended to be greater compared with that in the local control group $(-28.3 \% \pm$ $17.6 \%)$, though no significant differences were observed $(P=.28$ and $P=0.31$, respectively).

\section{DISCUSSION}

In this study, all pCASL scanning and TBF calculations were successfully performed in both the pretreatment and early treatment period. Although TBF measurement by pCASL in HNSCCs was recently reported to be feasible, ${ }^{19}$ there have been no reports on the use of this parameter in a clinical setting. Thus, the present study is the first to report the clinical usefulness of PCASL as a predictive factor in patients with HNSCC.

In regard to pretreatment $\mathrm{TBF}$, patients in the failure group included in our study exhibited lower values than patients in the local control group. In each primary lesion of the nasal or sinonasal cavity and oropharynx, the same tendency of lower pretreatment TBF was observed in the failure group compared with its respective local control group. A past reported review article introduced several studies that describe the use of pretreatment TBF measured by a dynamic contrast-enhanced perfusion technique for assessments of the prognosis of patients undergoing chemoradiotherapy. ${ }^{23}$ Most of these studies concluded that lower pretreatment TBF values indicated a poor prognosis. Our present findings showed the same trend as these previous reports.

With respect to the percentage change of TBF in the early treatment period, we observed that the patients in the failure group showed 1) significantly smaller percentage changes in the SD group, and 2) larger percentage changes in the PR group than the patients in the local control group. Truong et $\mathrm{al}^{14}$ reported that an initial increase in TBF in the early treatment period (2 weeks after the treatment started) in patients undergoing chemoradiotherapy predicted a good treatment result. The results of their report showed the same trend as that seen in the present study in regard to the patients who achieved SD status. In contrast, Surlan-Popovic et $\mathrm{al}^{15}$ and Gandhi et $\mathrm{al}^{16}$ reported that the follow-up TBF values during chemoradiotherapy were significantly lower in the patients with a good treatment response compared with those with a poor response, though more delayed timing was used in the study by Surlan-Popovic et $\mathrm{al}^{15}$ (the point of 40 Gy in a total of 65 Gy of radiation therapy) and the study by 
Gandhi et $\mathrm{al}^{16}$ (after 1 cycle of induction chemotherapy; 3-4 weeks after the treatment start) for the TBF measurement compared with the present study and the study by Truong et al. ${ }^{14}$ These descriptions of TBF decreases in patients with good treatment responses also showed the same trend as that seen in our present patients with PR.

In regard to these findings, we had several hypotheses. The good prognosis in patients with an increase in TBF has been attributed to an increase in tumor oxygenation, which affects the sensitivity of the treatment effect in radiation therapy. ${ }^{16,24} \mathrm{We}$ speculated that the initial TBF increase was caused by a decrease in the internal pressure of the tumor, which was derived from the initial treatment effect of cytotoxicity (ie, an increase in the extracellular space due to the destruction of tumor cells) in chemoradiotherapy, ${ }^{25-27}$ which resulted in the decrease in compression of intratumoral arteries. We suspected that such intratumoral changes reflected the initial TBF increase, probably observed as an initial treatment effect before the macroscopic TV reduction occurred. In contrast, the good prognosis in our patients with a TBF decrease reflected a progressed treatment effect, such as a decrease in an intratumoral arteriovenous shunt or a reduced volume of the vascular bed after chemoradiotherapy or damage to the endothelial cells of blood vessels by radiation therapy, ${ }^{28-30}$ and these changes in the tumor are likely to be observed at a more advanced period when the TV reduction has gradually occurred over the course of treatment compared with the initial TBF increase. However, in the present study population, these 2 trends of TBF increase or decrease were observed at the same time point during the treatment. The reason for this was probably related to the heterogeneity in the sensitivity of treatment response with tumors. ${ }^{31,32}$ We suspected that tumors with marked TV reduction even in the early treatment period would show a greater response to treatment compared with others, and we thus observed such a reversed TBF trend along with the change in the TV reduction rate at the same time point during treatment.

Several of our patients had local failure even though marked TV reduction was observed in the early treatment period in their cases. We suspected that this was because there will be intratumoral heterogeneity in treatment responses, such as that shown by a hypoxic lesion around the necrotic tissue or a deep area of the tumor with lower perfusion. ${ }^{19,33}$ Such lesions would continue to show little change of either TBF or TV, whereas other tumor lesions would show a volume reduction with marked TBF change.

Predicting the treatment result of local control or failure can provide useful information for decision-making regarding additional chemotherapy and the planning of earlier salvage surgery after the current chemoradiotherapy. For example, radiation therapy is often replanned due to a reduction in irradiated TV after an interim evaluation. The local distribution of TBF percentage change might be useful for replanning radiation therapy, as with ${ }^{18}$ F-FDG-PET. ${ }^{34}$ In the case of arterial infusion therapy, the dose distribution of drug infusion in each artery can also be adjusted, depending on the regional percentage change of TBF in each arterial territory.

The present study had several limitations. First, the distribution of the primary tumor sites leaned heavily toward the nasal or sinonasal cavity (24 of the 41 cases). To avoid a heterogeneous treatment plan, one of the study's inclusion criteria was treatment with an arterial infusion of cisplatin with concomitant radiation therapy. Systemic chemoradiotherapy tends to be the most commonly used treatment method for HNSCCs, especially in the oropharynx, but, with this therapy, it can sometimes be difficult to set the early treatment period due to the use of induction chemotherapy or to variation in the courses of chemotherapy drug administration. Second, no comparisons with other noninvasive parameters were conducted. The ADC measured from DWI has been widely investigated, and recent studies investigated the prediction of treatment response as well as the early detection of treatment response. ${ }^{23,25}$ Because TBF is a different biologic parameter than ADC, the combined use of both parameters could yield a more accurate prognosis. Further studies are needed to examine these points. Third, the patient number in the subgroup analysis based on SD and PR groups was quite small, and thus, the value of diagnostic accuracy from receiver operating characteristic analysis may not have much sufficient reliability. To address these problems, further analysis with greater numbers of patients will be considered necessary than in the current study. Fourth, other tumor environment factors, such as histologic differentiation, human papillomavirus status, and $\mathrm{T}$ stage, were not fully investigated as additional subgroup analysis. Because these factors may have a complex relationship with each other, the correlations among these factors and the identification of the factors that are related to the prognosis should be clarified in further studies.

\section{CONCLUSIONS}

The TBF values obtained by pCASL can provide useful information for the prediction of the local control: 1) higher pretreatment TBF values were suggested to indicate a local control, and 2) an increase in TBF in patients with a small TV reduction $(-30 \%$ or less) in their primary tumor at the early treatment period and a decrease in TBF in patients with a large TV reduction (more than $-30 \%$ ) were each suggested to indicate a local control. In particular, the use of the percentage change of TBF combined with the percentage change of TV had high diagnostic accuracy for predicting the local control.

Disclosures: Noriyuki Fujima—RELATED: Grant: The Ministry of Education, Culture, Sports, Science, and Technology, Japan (ID 25861047). Kohsuke Kudo-UNRELATED: Grants/Grants Pending: NEXT Program, * JSPS KAKENHI Grant*; Payment for Lectures (including service on speakers bureaus): GE Healthcare, Toshiba Medical Systems, Hitachi Medical Systems, Philips Medical Systems, Siemens Medical Systems, Bayer Healthcare. Hiroki Shirato-UNRELATED: Grants/Grants Pending: Hitachi,* Mitsubishi Heavy Industry, ${ }^{*}$ Shimadzu, ${ }^{*}$ Comments: For radiotherapy research; Patents (planned, pending or issued): Hitachi*; Royalties: Olympus. **Money paid to the institution.

\section{REFERENCES}

1. Homma A, Oridate N, Suzuki F, et al. Superselective high-dose cisplatin infusion with concomitant radiotherapy in patients with advanced cancer of the nasal cavity and paranasal sinuses: a single institution experience. Cancer 2009;115:4705-14 CrossRef Medline

2. Wong SJ, Harari PM, Garden AS, et al. Longitudinal Oncology Registry of Head and Neck Carcinoma (LORHAN): analysis of chemoradiation treatment approaches in the United States. Cancer 2011; 117:1679-86 CrossRef Medline

3. Kimata Y, Uchiyama K, Ebihara S, et al. Postoperative complications and functional results after total glossectomy with microvascular

AJNR Am J Neuroradiol 37:342-48 Feb 2016 www.ajnr.org 347 
reconstruction. Plast Reconstr Surg 2000;106:1028-35 CrossRef Medline

4. Eisenhauer EA, Therasse P, Bogaerts J, et al. New response evaluation criteria in solid tumours: revised RECIST guideline (version 1.1). Eur J Cancer 2009;45:228-47 CrossRef Medline

5. Cao Y, Popovtzer A, Li D, et al. Early prediction of outcome in advanced head-and-neck cancer based on tumor blood volume alterations during therapy: a prospective study. Int J Radiat Oncol Biol Phys 2008;72:1287-90 CrossRef Medline

6. Fujima N, Kudo K, Yoshida D, et al. Arterial spin labeling to determine tumor viability in head and neck cancer before and after treatment. J Magn Reson Imaging 2014;40:920-28 CrossRef Medline

7. Folkman J, Klagsbrun M. Angiogenic factors. Science 1987;235: 442-47 CrossRef Medline

8. Faggioni L, Neri E, Bartolozzi C. CT perfusion of head and neck tumors: how we do it. AJR Am J Roentgenol 2010;194:62-69 CrossRef Medline

9. Li XS, Fan HX, Zhu HX, et al. The value of perfusion CT in predicting the short-term response to synchronous radiochemotherapy for cervical squamous cancer. Eur Radiol 2012;22:617-24 CrossRef Medline

10. Agrawal S, Awasthi R, Singh A, et al. An exploratory study into the role of dynamic contrast-enhanced (DCE) MRI metrics as predictors of response in head and neck cancers. Clin Radiol 2012;67:e1-5 CrossRef Medline

11. Bisdas S, Medov L, Baghi M, et al. A comparison of tumour perfusion assessed by deconvolution-based analysis of dynamic contrast-enhanced CT and MR imaging in patients with squamous cell carcinoma of the upper aerodigestive tract. Eur Radiol 2008;18:843-50 CrossRef Medline

12. de Jongh FE, van Veen RN, Veltman SJ, et al. Weekly high-dose cisplatin is a feasible treatment option: analysis on prognostic factors for toxicity in 400 patients. Br J Cancer 2003;88:1199-206 CrossRef Medline

13. Daugaard G, Abildgaard U, Holstein-Rathlou NH, et al. Renal tubular function in patients treated with high-dose cisplatin. Clin Pharmacol Ther 1988;44:164-72 CrossRef Medline

14. Truong MT, Saito N, Ozonoff A, et al. Prediction of locoregional control in head and neck squamous cell carcinoma with serial CT perfusion during radiotherapy. AJNR Am J Neuroradiol 2011;32: 1195-201 CrossRef Medline

15. Surlan-Popovic K, Bisdas S, Rumboldt Z, et al. Changes in perfusion CT of advanced squamous cell carcinoma of the head and neck treated during the course of concomitant chemoradiotherapy. AJNR Am J Neuroradiol 2010;31:570-75 CrossRef Medline

16. Gandhi D, Chepeha DB, Miller T, et al. Correlation between initial and early follow-up CT perfusion parameters with endoscopic tumor response in patients with advanced squamous cell carcinomas of the oropharynx treated with organ-preservation therapy. AJNR Am J Neuroradiol 2006;27:101-06 Medline

17. Lanzman RS, Robson PM, Sun MR, et al. Arterial spin-labeling MR imaging of renal masses: correlation with histopathologic findings. Radiology 2012;265:799-808 CrossRef Medline

18. Binnewijzend MA, Kuijer JP, Benedictus MR, et al. Cerebral blood flow measured with 3D pseudocontinuous arterial spin-labeling MR imaging in Alzheimer disease and mild cognitive impairment: a marker for disease severity. Radiology 2013;267:221-30 CrossRef Medline

19. Fujima N, Kudo K, Tsukahara A, et al. Measurement of tumor blood flow in head and neck squamous cell carcinoma by pseudo-continuous arterial spin labeling: comparison with dynamic contrast-enhanced MRI. J Magn Reson Imaging 2015;41:983-91 CrossRef Medline

20. Wang Z, Aguirre GK, Rao H, et al. Empirical optimization of ASL data analysis using an ASL data processing toolbox: ASLtbx. Magn Reson Imaging 2008;26:261-69 CrossRef Medline

21. van Osch MJ, Teeuwisse WM, van Walderveen MA, et al. Can arterial spin labeling detect white matter perfusion signal? Magn Reson Med 2009;62:165-73 CrossRef Medline

22. Wheeler RH, Ziessman HA, Medvec BR, et al. Tumor blood flow and systemic shunting in patients receiving intraarterial chemotherapy for head and neck cancer. Cancer Res 1986;46:4200-04 Medline

23. Srinivasan A, Mohan S, Mukherji SK. Biologic imaging of head and neck cancer: the present and the future. AJNR Am J Neuroradiol 2012;33:586-94 CrossRef Medline

24. Bhatnagar P, Subesinghe M, Patel C, et al. Functional imaging for radiation treatment planning, response assessment, and adaptive therapy in head and neck cancer. Radiographics 2013;33:1909-29 CrossRef Medline

25. King $\mathrm{AD}$, Chow KK, $\mathrm{Yu} \mathrm{KH}$, et al. Head and neck squamous cell carcinoma: diagnostic performance of diffusion-weighted MR imaging for the prediction of treatment response. Radiology 2013;266: 531-38 CrossRef Medline

26. Kauppinen RA. Monitoring cytotoxic tumour treatment response by diffusion magnetic resonance imaging and proton spectroscopy. NMR Biomed 2002;15:6-17 CrossRef Medline

27. Berrak S, Chawla S, Kim S, et al. Diffusion weighted imaging in predicting progression free survival in patients with squamous cell carcinomas of the head and neck treated with induction chemotherapy. Acad Radiol 2011;18:1225-32 CrossRef Medline

28. Zwelling LA, Kohn KW. Mechanism of action of cis-dichlorodiammineplatinum(II). Cancer Treat Rep 1979;63:1439-44 Medline

29. Leek RD, Landers RJ, Harris AL, et al. Necrosis correlates with high vascular density and focal macrophage infiltration in invasive carcinoma of the breast. Br J Cancer 1999;79:991-95 CrossRef Medline

30. Martin DF, Fischer JJ. Radiation sensitivity of cultured rabbit aortic endothelial cells. Int J Radiat Oncol Biol Phys 1984;10:1903-06 CrossRef Medline

31. Kojima H, Endo K, Moriyama H, et al. Abrogation of mitochondrial cytochrome $\mathrm{C}$ release and caspase- 3 activation in acquired multidrug resistance. J Biol Chem 1998;273:16647-50 CrossRef Medline

32. Godwin AK, Meister A, O’Dwyer PJ, et al. High resistance to cisplatin in human ovarian cancer cell lines is associated with marked increase of glutathione synthesis. Proc Natl Acad Sci U S A 1992;89: 3070-74 CrossRef Medline

33. Evans SM, Du KL, Chalian AA, et al. Patterns and levels of hypoxia in head and neck squamous cell carcinomas and their relationship to patient outcome. Int J Radiat Oncol Biol Phys 2007;69:1024-31 CrossRef Medline

34. Delouya G, Igidbashian L, Houle A, et al. ${ }^{18}$ F-FDG-PET imaging in radiotherapy tumor volume delineation in treatment of head and neck cancer. Radiother Oncol 2011;101:362-68 CrossRef Medline 